\title{
Can Medical Research Do Its Job?
}

\author{
Jay Pravda ${ }^{1}$ (D)
}

Published online: 2 October 2017

(C) The Author(s) 2017. This article is an open access publication

\section{Essay}

We've heard it often enough. A medical disease researcher will announce some important breakthrough; never to be heard from again. So, what's the problem? Why can't medical science figure out the cause of diseases such as inflammatory bowel disease (ulcerative colitis and Crohn's disease) sepsis and neurodegenerative disease (Alzheimer's, Parkinson's, and multiple sclerosis) to name a few?

Heaven knows we've been working on them long enough and billions of dollars have been poured into research over the past 50 years to no avail. Despite having the most advanced technology and the smartest and dedicated researchers working on the problem, we are no closer to finding the cause or a cure for these diseases that afflict more and more people every year. In fact, it's hard to find someone whose life hasn't been touched in some horrendous way by these incurable afflictions that also include autism, cancer, and systemic lupus erythematosus among others.

Despite the lack of progress, we soldier on; going back to the laboratory and doing more experiments in the hope that one fortunate happenstance will provide the breakthrough that will lead to a cure. Although serendipity may work well with a pot-luck party, it's not the way to approach medical research if we want to find cures before the burden of disease becomes so overwhelming; financially, emotionally, politically, and socially overwhelming that we simply give up hope in our ability to conquer these diseases.

Jay Pravda

jay.pravda@therashock.com

1 Therashock LLC, Wilmington, DE, USA

Although we have made great strides in building up our medical research infrastructure with investments in technology and medical scientist training programs, without a theory of disease to guide future experiments we are simply groping in the dark when it comes to medical research. To illustrate how important a theory of disease can be; the germ theory of disease paved the way for advances in immunization, pasteurization, antibiotics, public health, and surgery. Most of us (including me) would not be here today if not for this simple theory.

But who comes up with these theories? With over 26 million research publications in the National Library of Medicine's database (PubMed) [1], laboratory based researchers don't have enough hours in the day to do the timeconsuming relational data mining needed to understand how and where their results fit in and propose falsifiable theories of disease that arise from insight gained by studying little known areas of medicine such as redox biochemistry, bioenergetics and redox biology in addition to emerging sources of information such as "big data" [2].

Theoretical biology and medicine is the missing piece of the puzzle. Laboratory-based researchers are trained to design and carry out experiments not to develop new theories of disease that can lead to a cure. Medical theorists are needed to propose new theories of disease and help guide laboratory research along a focused path of discovery that leads to the cause of diseases and their cures. Medicine needs to realize that "different minds are suited to different aspects of research and society as-a-whole benefits from the interplay of theorists and experimentalists" [3]. Theorists provide the creativity without which medical research cannot flourish and advance. Without guiding theories of disease, medical research into causation is haphazard and wasteful; eventually settling into decades of stagnation that we see today, forcing clinicians to treat sick patients using algorithms or consensus guidelines in 
an effort to plug the gap in our knowledge of pathogenesis that research is meant to supply.

Physicists realized the value of theory in the early 20th century and today we have the separate branches of theoretical and experimental physics that continue to advance the science into unimaginable realms. However, medicine lags far behind. We have the finest experimental research laboratories focused on producing data, which provide a small piece of the puzzle to the cause of disease but without a wider integration into all previously published results guided by a plausible theory of disease the data just sits on PubMed's website, waiting for a medical theorist to put the pieces all together and predict what the cause actually is.

Unfortunately, no graduate programs in theoretical biology and medicine exist in the United States. Educational grants for pilot programs must be provided and policy changes enacted to address this shortcoming starting with graduate biomedical training programs in theoretical biology and medicine that emphasize a patho-mechanistic model of medical education as an alternative to the current patient care approach. Creative divergent thinking and not rote memorization should be the basis of this new curriculum. Faculty positions should be made available for these theoretical medical specialists whose needed expertise should be brought to bear on the serious diseases facing society today. Additionally, theoretical biology and medicine should be promoted with equal standing as career options for undergraduates along with more traditional laboratory based research careers.

Without a disruptive change in medical education and the biomedical research landscape the burden of disease will continue to prey upon our youngest, our best, our brightest and the most vulnerable members of our society. We don't have a choice. The status quo is simply not working.

Open Access This article is distributed under the terms of the Creative Commons Attribution 4.0 International License (http:// creativecommons.org/licenses/by/4.0/), which permits unrestricted use, distribution, and reproduction in any medium, provided you give appropriate credit to the original author(s) and the source, provide a link to the Creative Commons license, and indicate if changes were made.

\section{References}

1. PubMed Help [Internet]. Bethesda (MD): National Center for Biotechnology Information (US); 2005-. PubMed Help. [Updated 2017 May 25] Available from: https://www.ncbi.nlm.nih.gov/ books/NBK3827/.

2. Lee $\mathrm{CH}$, Yoon HJ. Medical big data: promise and challenges. Kidney Res Clin Pract. 2017;36:3.

3. Huszagh VA, Infante JP. The hypothetical way of progress. Nature. 1989;338(6211):109. 